Pacific Journal of Mathematic 


\title{
PM-NORMALITY AND THE INSERTION OF A CONTINUOUS FUNCTION
}

\author{
ERnest P. LANE
}

\begin{abstract}
Spaces in which each regular closed subset is an intersection of a sequence of closed neighborhoods are investigated. This property is shown to be equivalent to each of the following: Each regular closed subset is a zero set of a continuous function. Each normal lower semicontinuous function defined on the space is a limit of an increasing sequence of continuous functions. The space satisfies the strong $C$ insertion property for normal semicontinuous functions. Separation properties of $X \times I$, which are weaker than normality, are related to the insertion of a continuous function between two comparable functions defined on $X$.
\end{abstract}

In greater detail, the following conditions are shown to be equivalent: (i) Each regular closed subset is an intersection of a sequence of closed neighborhoods. (I.e., each regular closed subset is a regular $G_{\dot{0}}$.) (ii) Each regular closed subset is a zero set of a continuous function. (iii) For each normal lower semicontinuous function $f$ defined on the space and for each real number $r,\{x \in X: f(x) \leqq r\}$ is a zero set of a continuous function. (iv) If $g$ and $f$ are real valued functions defined on $X$ such that $g$ is normal upper semicontinuous, $f$ is normal lower semicontinuous and $g \leqq f$, then there exists a continuous function $h$ defined on $X$ such that $g \leqq h \leqq f$ and such that for any point $x$ for which $g(x)<f(x)$, then $g(x)<h(x)<f(x)$. (v) Each normal lower semicontinuous function on $X$ is a limit of an increasing sequence of continuous functions. Any space that satisfies one of these equivalent conditions is a weak $c b$-space and has disjoint regular closed subsets separated by disjoint open sets.

1. pm-normal spaces. A subset $B$ of a space $X$ is a regular $G_{\delta}$ in case $B$ is an intersection of a sequence of closed sets whose interiors contain $B$. A space is mildly normal in case disjoint regular closed subsets are separated by disjoint open sets. Since it turns out that any space where each regular closed subset is a regular $G_{\delta}$ is mildly normal, as will be noted below, it is convenient to make the following definition: A topological space $X$ is pm-normal (for perfectly mildly normal) in case each regular closed subset of $X$ is a regular $G_{j}$.

The abbreviations lsc [respectively, usc] for lower [respectively, upper] semicontinuous and nlsc [respectively, nusc] for normal lower [respectively, normal upper] semicontinuous are used throughout the 
remainder of the paper. The upper and lower limit functions of $f$, which are denoted by $f^{*}$ and $f_{*}$, respectively, are defined as follows. For each $x$ in $X$, let

$$
f^{*}(x)=\inf \left\{\sup _{y \in N} f(y): N \text { is a neighborhood of } x\right\}
$$

and

$$
f_{*}(x)=\sup \left\{\inf _{y \in N} f(y): N \text { is a neighborhood of } x\right\} \text {. }
$$

The function $f$ is nlsc if and only if $f=\left(f^{*}\right)_{*}$ and $f$ is nusc if and only if $f=\left(f_{*}\right)^{*}$.

THEOREM 1.1. Consider the following conditions:

(i) A space $X$ is pm-normal.

(ii) Each regular closed subset of $X$ is a zero set.

(iii) For each nlsc function $f$ and for each real number $r$, $\{x \in X: f(x) \leqq r\}$ is a zero set.

(iv) Each nlsc function defined on the space is a limit of an increasing sequence of continuous functions.

(v) A space $X$ is mildly normal.

(vi) Each countable regular open cover of $X$ admits a locally finite partition of unity subordinate to it.

(vii) A space $X$ is weak $c b$.

Then (i), (ii), (iii), and (iv) are equivalent, (i) implies (v), (i) implies (vi), and (vi) implies (vii).

Proof. (i) $\Rightarrow$ (v). If $F$ and $K$ are disjoint regular closed subsets, then by (i), $F=\bigcap_{n=1}^{\infty} A_{n}$ and $K=\bigcap_{n=1}^{\infty} B_{n}$ where $A_{n}$ and $B_{n}$ are closed and $F \subset \inf A_{n}$ and $K \subset \operatorname{int} B_{n}$ for each $n$. Since

$\left[\cap_{n=1}^{\infty} \mathrm{cl}\left(\right.\right.$ int $\left.\left.A_{n}\right)\right] \cap K=\varnothing$ and $\left[\bigcap_{n=1}^{\infty} \operatorname{cl}\left(\right.\right.$ int $\left.\left.B_{n}\right)\right] \cap F=\varnothing$, it follows from Lemma 2.6 of Zenor [16] that there exists an open set $G$ that contains $F$ such that (cl $G) \cap K=\varnothing$. Thus $X$ is mildly normal.

(i) $\Rightarrow$ (ii). If $D$ is a regular closed set, then by (i), $D=\bigcap_{n=1}^{\infty} E_{n}$ where each $E_{n}$ is a closed set such that $D \subset \operatorname{int} E_{n}$. Since $D$ and $X$ - int $E_{n}$ are disjoint regular closed sets and since (i) implies that $X$ is mildly normal by the above proof, it follows from Corollary 3.3 of Lane [6] that $D$ and $X$-int $E_{n}$ are completely separated. Since there exist zero sets $Z_{n}$ such that $D \subset Z_{n} \subset \inf E_{n}$ and since $D=\bigcap_{n=1}^{\infty} Z_{n}$, then $D$ is a zero set.

(ii) $\Rightarrow$ (iii). If $f$ is nlsc and if $r$ is a real number, then by Theorem 3.2 of Dilworth [1], $\left\{x \in X: f(x)<r+2^{-n}\right\}$ is a union of regular closed sets for each natural number $n$. Thus $\operatorname{cl}\left\{x \in X: f(x)<r+2^{-n}\right\}$ is a regular closed set; since

$$
\{x \in X: f(x) \leqq r\}=\bigcap_{n=1}^{\infty} \operatorname{cl}\left\{x \in X: f(x)<r+2^{-n}\right\},
$$


it follows from (ii) that $\{x \in X: f(x) \leqq r\}$ is a countable intersection of zero sets. Thus $\{x \in X: f(x) \leqq r\}$ is a zero set.

If condition (iii) is satisfied, Tong's proof [15] that each lsc function on a perfectly normal space is a limit of an increasing sequence of continuous functions is trivially modified to show that (iv) is valid. The proof that (iv) implies (i) is elementary and is omitted.

(i) $\Rightarrow$ (vi). If (i) is satisfied, then any countable regular open cover of $X$ is a countable cozero cover of $X$. By Lemma 5.2.4 of Engelking [3], this cover has a countable locally finite refinement consisting of cozero sets. By Theorem 1.2 of Morita [13], this locally finite cozero cover has a locally finite partition of unity subordinate to it.

That condition (vii) is an immediate consequence of condition (iv) follows from Theorem 3.1 of Mack and Johnson [11].

It is straightforward to show that any open subspace of a pmnormal space is pm-normal. Since any closed subset of a perfectly normal space is a zero set and hence a regular $G_{j}$ set, it follows that a perfectly normal space is pm-normal. But a $c b$-space need not be pm-normal. The closed ordinal space $[0, \Omega]$ satisfies conditions $(v)$ and (vi) of the above theorem but is not a pm-normal space. The following example shows that a pm-normal space need not be perfectly normal: Let $X$ consist of concentric circles $C_{1}$ and $C_{2}$. A basic open set about a point $x$ on the inner circle $C_{1}$ consists of an arc on $C_{1}$ centered at $x$ together with the projection of this arc on the outer circle $C_{2}$; a basic open set about a point $y$ on the outer circle is an arc on $C_{2}$ centered at $y$. If $F$ is a closed subset of $X$ and if $y$ is in $F \cap C_{2}$, then the radial projection $x$ of $y$ onto $C_{1}$ must be in $F$. If $F$ is a regular closed subset of $X$ and if $x$ is in $F \cap C_{1}$, then the radial projection $y$ of $x$ onto $C_{2}$ must be in $F$. It is easy to see that a closed subset of $X$ need not be a zero set while each regular closed subset of $X$ is a zero set. Consequently $X$ is pm-normal but not perfectly normal.

2. Strong $C$ insertion. It is convenient to use the terminology of Lane [7] throughout the remainder of the paper. The following definitions are repeated here for convenience. A property $P$ defined relative to a real valued function on a topological space is a $C$ property provided any constant function has property $P$ and provided the sum of a function with property $P$ and a continuous function also has property $P$. If $P_{1}$ and $P_{2}$ are $C$ properties, then: (i) A space $X$ has the weak $C$ insertion [respectively, $C$ insertion] property for $\left(P_{1}, P_{2}\right)$ if and only if for any functions $g$ and $f$ on $X$ such that $g \leqq f$ [respectively, $g<f], g$ has property $P_{1}$, and $f$ has property $P_{2}$, then 
there exists a continuous function $h$ on $X$ such that $g \leqq h \leqq f$ [respectively, $g<h<f$ ]. (ii) A space $X$ has the strong $C$ insertion property for $\left(P_{1}, P_{2}\right)$ if and only if for any functions $g$ and $f$ on $X$ such that $g \leqq f, g$ has property $P_{1}$, and $f$ has property $P_{2}$, then there exists a continuous function $h$ on $X$ such that $g \leqq h \leqq f$ and such that for any $x$ in $X$ for which $g(x)<f(x)$ then $g(x)<h(x)<$ $f(x)$. The following simple relationship between these concepts is established.

Proposition 2.1. Let $P_{1}$ and $P_{2}$ be $C$ properties and assume that the space $X$ satisfied the weak $C$ insertion property for $\left(P_{1}, P_{2}\right)$. The space $X$ satisfies the strong $C$ insertion property for $\left(P_{1}, P_{2}\right)$ if and only if $X$ satisfies the strong $C$ insertion property for $\left(P_{1}\right.$, continuous) and for (continuous, $P_{2}$ ).

Proof. Assume that $X$ satisfies the strong $C$ insertion property for $\left(P_{1}\right.$, continuous) and for (continuous, $\left.P_{2}\right)$. If $g$ and $f$ are functions on $X$ such that $g \leqq f, g$ satisfies property $P_{1}$, and $f$ satisfies property $P_{2}$, then since $X$ satisfies the weak $C$ insertion property for $\left(P_{1}, P_{2}\right)$ there is a continuous function $k$ on $X$ such that $g \leqq k \leqq f$. Also, by hypothesis there exist continuous functions $h_{1}$ and $h_{2}$ on $X$ such that $g \leqq h_{1} \leqq k$ and if $g(x)<k(x)$ then $g(x)<h_{1}(x)<k(x)$ and such that $k \leqq h_{2} \leqq f$ and if $k(x)<f(x)$ then $k(x)<h_{2}(x)<f(x)$. If a function $h$ is defined by $h(x)=\left(h_{2}(x)+h_{1}(x)\right) / 2$, then $h$ is continuous, $g \leqq h \leqq f$, and if $g(x)<f(x)$ then $g(x)<h(x)<f(x)$. Hence $X$ satisfies the strong $C$ insertion property for $\left(P_{1}, P_{2}\right)$. The converse is obvious since any continuous function must satisfy both properties $P_{1}$ and $P_{2}$.

The following result was announced in Lane [5].

THEOREM 2.2. The following conditions are equivalent:

(i) The space is pm-normal.

(ii) The space has the strong $C$ insertion property for (nusc, continuous) [respectively, (continuous, nlsc)].

(iii) The space has the strong $C$ insertion property for (nusc, $n l s c)$.

Proof. In order to see that (i) implies (ii), let $g$ and $f$ be functions defined on a pm-normal space $X$ such that $g \leqq f, g$ is nusc, and $f$ is continuous. Since $f-g$ is nlse, it follows from Thorem 1.1 that

$$
A=\{x \in X:(f-g)(x)=0\}
$$

is a zero set. Thus there exists a continuous function $p$ mapping $X$ into $[0,1]$ such that $p^{-1}(0)=A$. Since $X-A$ is a cozero subset 
of the weak $c b$-space $X$, it follows from Proposition 3.2 of Mack and Johnson [11] that $X-A$ is a weak $c b$-space. Thus, as noted in Lane [7], the space $X-A$ has the $C$ insertion property for (nusc, continuous). Thus there is a continuous function $u$ defined on $X-A$ such that $g(x)<u(x)<f(x)$ for all $x$ in $X-A$. Let $v$ be a function defined on $X$ by

$$
\begin{array}{ll}
v(x)=0 & \text { if } x \in A \\
v(x)=\frac{p(x)}{1+|f(x)-u(x)|}(f(x)-u(x)) & \text { if } \quad x \in X-A .
\end{array}
$$

Then $v$ is a continuous function defined on $X$. If $h=f-v$, then $h$ is a continuous function on $X, g \leqq h \leqq f$ and if $g(x)<f(x)$, then $g(x)<h(x)<f(x)$. Thus $X$ satisfies the strong $C$ insertion property for (nusc, continuous). (This proof uses a technique due to Michael [12]. It is also possible to construct a proof of this implication based on Theorem 3.1 of Lane [7].)

Assume that $X$ satisfies condition (ii). If $F$ is a regular closed subset of $X$, and if $f=1$ on $X, g=1$ on $F$ and $g=0$ on $X-F$, then, $g \leqq f, g$ is nusc, and $f$ is continuous. By (ii) there is a continuous function $h$ on $X$ such that $g \leqq h \leqq f$ and $0<h<1$ on $X-F$. Since $F=\bigcap_{n=1}^{\infty}\left\{x \in X: h(x) \geqq 1-n^{-1}\right\}, F$ is a zero set. Thus $X$ is mildly normal and consequently $X$ satisfies the weak $C$ insertion property for (nusc, nlsc) (by Corollary 2 of Lane [8]). In view of Proposition 2.1, condition (iii) is satisfied. The proof that (iii) implies (i) is straightforward and is omitted.

Michael [12] proved that a space satisfies the strong $C$ insertion property for (usc, lsc) if and only if the space is perfectly normal. This result was obtained later as a corollary to Theorem 3.1 of Lane [7]. It is a trivial observation that strong $C$ insertion for (usc, lsc) and strong $C$ insertion for (usc, continuous) [respectively, (continuous, lsc)] are equivalent in a perfectly normal space. It therefore should have been observed that Corollary 3.3 on page 189 of Lane [7] is an immediate consequence of Michael's result. It appears, however, that the observation made in Proposition 2.1 is useful in the proof of condition (iii) of Theorem 2.2. In the situation where $f$ is nlsc and $g$ is nusc, neither the technique used by Michael [12] nor Theorem 3.1 of Lane [7] applies since $f-g$ need not be nlsc. It was observed in Corollary 3.3 on page 188 of Lane [7] that a space $X$ satisfies the strong $C$ insertion property for (usc, nlsc) [respectively, (nusc, lsc)] if and only if $X$ is almost normal and if each closed subset of $X$ is an intersection of a sequence of regular open sets. By Corollary 2 of Zenor [16], these properties of $X$ are seen to be equivalent to $X$ is perfectly normal. Hence this result is also a corollary of Michael's result. In summary, the strong $C$ insertion properties for (i) (usc, 
Isc); (ii) (usc, nlsc); (iii) (usc, continuous) are equivalent.

3. $C$ insertion and $X \times I$. The following equivalences are known: (i) The space $X$ satisfies the $C$ insertion property for (usc, lsc) if and only if $X \times I$ is normal. (ii) The space $X$ satisfies the $C$ insertion property for (usc, continuous) [respectively, (continuous, lsc)] if and only if $X \times I$ is $\delta$-normally separated. (iii) The space $X$ satisfies the $C$ insertion property for (nusc, continuous) [respectively, (continuous, nlsc)] if and only if $X \times I$ is weakly $\delta$-normally separated. The equivalence (i) was proved independently by Dowker [2] and by Katětov [3] by proving that each of the two properties is equivalent to $X$ is normal and countably paracompact. Condition (ii) follows from Mack [9] and [10] by observing that each of the two properties is equivalent to $X$ is a $c b$-space. Similarly, (iii) follows from Mack [10] and from Mack and Johnson [11] by observing that each of the properties is equivalent to $X$ is a weak $c b$-space. The following results are an attempt to understand the relationship between $C$ insertion for the space $X$ and separation properties for $X \times I$ by discovering a direct relationship.

LeMma 3.1. If $f$ is a function from $X$ into the interior of $I$, let

$$
L_{f}=\{(x, t) \in X \times I: 0 \leqq t \leqq f(x)\}
$$

and

$$
U_{f}=\{(x, t) \in X \times I: f(x) \leqq t \leqq 1\}
$$

(i) If $f$ is continuous, then $L_{f}$ and $U_{f}$ are zero sets in $X \times I$.

(ii) If $f$ is lsc [respectively, usc], then $U_{f}$ [respectively, $\left.L_{f}\right]$ is closed in $X \times I$.

(iii) If $f$ is nlsc [respectively, nusc], then $U_{f}$ [respectively, $\left.L_{f}\right]$ is regular closed in $X \times I$.

The proof of the lemma, which is elementary, is omitted. This observation and certain results concerning weak $C$ insertion properties of the space $X$ are utilized to obtain the following propositions.

THEOREM 3.2. If $X \times I$ is mildly normal, then $X$ satisfies the $C$ insertion property for (nusc, nlsc).

Proof. If $g$ and $f$ are functions on $X$ such that $g<f, g$ is nusc, $f$ is nlsc, let

$$
\tilde{f}=\left[f(1+|f|)^{-1}+1\right] / 2
$$




$$
\widetilde{g}=\left[g(1+|g|)^{-1}+1\right] / 2 .
$$

Then $\widetilde{g}$ and $\tilde{f}$ map $X$ into $(0,1), \widetilde{g}<\widetilde{f}, \widetilde{g}$ is nusc, and $\tilde{f}$ is nlsc. By Lemma 3.1, $L_{\tilde{g}}$ and $U_{\widetilde{f}}$ are regular closed subsets of $X \times I$. Since $X \times I$ is mildly normal and since $L_{\tilde{g}}$ and $U_{\tilde{f}}$ are disjoint, these sets are completely separated. If $F$ is a continuous function from $X \times I$ into $I$ such that $F=0$ on $L_{\tilde{g}}$ and $F=1$ on $U_{\tilde{f}}$, define a function $\tilde{b}$ from $X$ into $(0,1)$ as follows:

$$
\widetilde{b}(x)=\sup \{t \in I: F(x, t) \leqq 1 / 2\} .
$$

Then $F(x, \widetilde{b}(x))=1 / 2, \widetilde{g}<\widetilde{b}<\widetilde{f}$, and since $\widetilde{b}$ is the upper boundary function of the closed subset

$$
\{(x, t) \in X \times I: F(x, t) \leqq 1 / 2\},
$$

$\widetilde{b}$ is usc.

If a function $\tilde{k}$ from $X$ into $I$ is defined by

$$
\widetilde{k}(x)=\inf \{t: F(x, t) \geqq 1 / 2\}
$$

then, as above, $\tilde{k}$ is lsc. Also, $\widetilde{g}<\tilde{k} \leqq \tilde{b}<\tilde{f}$. Since $\tilde{k}$ is lsc, $\tilde{b}$ is usc, and $\widetilde{k} \leqq \widetilde{b}$, it follows that $\widetilde{k} \leqq\left((\widetilde{k})^{*}\right)_{*} \leqq \widetilde{b}$. Since $\left(\left(\left((\widetilde{k})^{*}\right)_{*}\right)^{*}\right)_{*}=$ $\left((\widetilde{k})^{*}\right)_{*}$, the function $\widetilde{d}$ defined by $=\left((\widetilde{\bar{d}})^{*}\right)_{*}$ is nlsc. And $\widetilde{g}<\widetilde{d}<\widetilde{f}$. Since $-\widetilde{d}$ is nusc, $-\widetilde{g}$ is nlsc, and since $-\widetilde{d}<-\widetilde{g}$, then by exactly the same argument as used above, there is a nlsc function $-\widetilde{p}$ defined on $X$ such that $-\widetilde{d}<-\widetilde{p}<-\widetilde{g}$. Since $X \times I$ mildly normal immediately implies that $X$ is mildly normal, then $X$ satisfies the weak $C$ insertion property for (nusc, nlsc) by Corollary 2 in Lane [8]. Thus there is a continuous function $\widetilde{h}$ on $X$ such that $\widetilde{p} \leqq \widetilde{h} \leqq \widetilde{d}$. If

$$
h(x)=(2 h(x)-1) /(1-|2 h(x)-1|),
$$

then $h$ is a continuous function on $X$ with $g<h<f$. Thus $X$ has the $C$ insertion property for (nusc, nlsc).

It is straightforward to see that if $X$ satisfies the $C$ insertion property for (nusc, nlsc), then $X$ is mildly normal and a weak $c b$ space. It would be interesting to know if the converse of this is valid or if the converse of Theorem 3.2 is true. Shchepin [14] gave an example of a mildly normal space $X$ such that $X \times I$ is not mildly normal.

Since an almost normal space satisfies the weak $C$ insertion property for (usc, nlsc) [respectively, (nusc, lsc)], it is possible to use the result of Lemma 3.1 and the technique of the proof of Theorem 3.2 to establish the following result. The details of the proof are entirely analogous and are omitted. 
THEOREM 3.3. If $X \times I$ is almost normal, then $X$ satisfies the $C$ insertion property for (usc, nlsc) [respectively, (nusc, lsc)].

The author is indebted to the referee for suggestions that led to the improvement of the paper. In particular, the proof of Theorem 3.2 was significantly simplified.

Added in proof. Reference should have been given to Robert L. Blair's paper "Spaces In Which Special Sets Are $z$-Embedded," Canad. J. Math., 28 (1976), 673-690; the concept of a pm-normal space was investigated and was termed an $O_{z}$ space.

\section{REFERENCES}

1. R. P. Dilworth, The normal completion of the lattice of continuous functions, Trans. Amer. Math. Soc., 68 (1950), 427-438.

2. C. H. Dowker, On countably paracompact spaces, Canad. J. Math., 3 (1951), 219-224.

3. R. Engleking, General Topology, Polish Scientific Publishers, Warsaw, Poland, 1977.

4. M. Katětov, On real-valued functions in topological spaces, Fund. Math., 38 (1951), 85-91.

5. E. P. Lane, Insertion of a continuous function, Notices Amer. Math. Soc., (October, 1977).

6. - Insertion of continuous functions, Glasnik Mat. Series III, 6 (25) (1971), $165-171$.

7. - Insertion of a continuous function, Pacific J. Math., 66 (1976), 181-190.

8. - A sufficient condition for the insertion of a continuous function, Proc. Amer. Math. Soc., 49 (1975), 90-94.

9. J. E. Mack, On a class of countably paracompact spaces, Proc. Amer. Math. Soc., 16 (1965), 467-472.

10. Countable paracompactness and weak normality properties, Trans. Amer. Math. Soc., 148 (1970), 265-272.

11. J. E. Mack and D. G. Johnson, The Dedekind completion of $C(X)$, Pacific J. Math., 20 (1967), 231-243.

12. E. Michael, Continuous selections I, Ann. of Math., 63 (1956), 361-382.

13. K. Morita, Paracompactness and product spaces, Fund. Math., 50 (1961), 223-236.

14. E. V. Shchepin, Real functions and near-normal spaces, Sibirskii Mate. Zhurnal,

13 (1972), 1182-1195.

15. H. Tong, Some characterizations of normal and perfectly normal spaces, Duke Math. J., 19 (1952), 289-292.

16. P. Zenor, On countable paracompactness and normality, Prace Math., 13 (1969), 23-32.

Received August 23, 1978 and in revised form November 20, 1978. This work was supported by a summer reseach grant from Appalachian State University.

Appalachian State University

BOONE, NC 28608 


\section{PACIFIC JOURNAL OF MATHEMATICS}

\section{EDITORS}

DoNALD BABBITT (Managing Editor)

University of California

Los Angeles, California 90024

HUGo Rossi

University of Utah

Salt Lake City, UT 84112

C. C. MOORE and ANDREW OGG

University of California

Berkeley, CA 94720
J. DUGUNDJI

Department of Mathematics University of Southern Californı Los Angeles, California 90007

R. Finn and J. Milgram Stanford University Stanford, California 94305

\section{ASSOCIATE EDITORS}

E. F. BECKENBACH

B. H. Neumann
F. WOLF

K. YOSHIDA

\section{SUPPORTING INSTITUTIONS}

UNIVERSITY OF BRITISH COLUMBIA CALIFORNIA INSTITUTE OF TECHNOLOGY UNIVERSITY OF CALIFORNIA MONTANA STATE UNIVERSITY UNIVERSITY OF NEVADA, RENO NEW MEXICO STATE UNIVERSITY OREGON STATE UNIVERSITY UNIVERSITY OF OREGON
UNIVERSITY OF SOUTHERN CALIFORNIA STANFORD UNIVERSITY UNIVERSITY OF HAWAII UNIVERSITY OF TOKYO UNIVERSITY OF UTAH WASHINGTON STATE UNIVERSITY UNIVERSITY OF WASHINGTON 


\section{Pacific Journal of Mathematics}

Vol. 82, No. $1 \quad$ January, 1979

Werner Bäni, Subspaces of positive definite inner product spaces of countable dimension ...................................... 1

Marilyn Breen, The dimension of the kernel of a planar set..............

Kenneth Alfred Byrd, Right self-injective rings whose essential right ideals

are two-sided

Patrick Cousot and Radhia Cousot, Constructive versions of Tarski's fixed

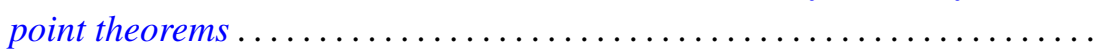

Ralph S. Freese, William A. Lampe and Walter Fuller Taylor, Congruence lattices of algebras of fixed similarity type. $I \ldots \ldots \ldots \ldots \ldots \ldots \ldots$

Cameron Gordon and Richard A. Litherland, On a theorem of Murasugi .....

Mauricio A. Gutiérrez, Concordance and homotopy. I. Fundamental

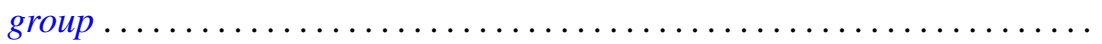

Richard I. Hartley, Metabelian representations of knot groups .............

Ted Hurley, Intersections of terms of polycentral series of free groups and free

Lie algebras ........................................

Roy Andrew Johnson, Some relationships between measures ............ 117

Oldřich Kowalski, On unitary automorphisms of solvable Lie algebras .......

Kee Yuen Lam, $K O$-equivalences and existence of nonsingular bilinear

maps...................................................

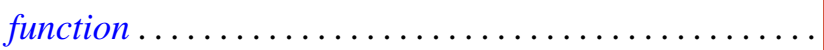

Robert A. Messer and Alden H. Wright, Embedding open 3-manifolds in compact 3-manifolds ............................

Gerald Ira Myerson, A combinatorial problem in finite fields. I . .

James Nelson, Jr. and Mohan S. Putcha, Word equations in a band of paths.

Baburao Govindrao Pachpatte and S. M. Singare, Discrete generalized Gronwall inequalities in three independent variables . .

William Lindall Paschke and Norberto Salinas, $C^{*}$-algebras associated with free products of groups ........................

Bruce Reznick, Banach spaces with polynomial norms ....

David Rusin, What is the probability that two elements of a finite group

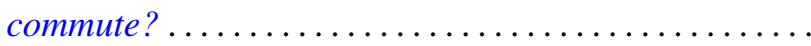

M. Shafii-Mousavi and Zbigniew Zielezny, On hypoelliptic differential operators of constant strength ...

Joseph Gail Stampfli, On selfadjoint derivation ranges .... . . .

Robert Charles Thompson, The case of equality in the matrix-valued triangle

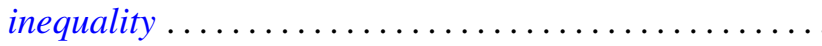

Marie Angela Vitulli, The obstruction of the formal moduli space in the negatively graded case. 